\title{
Constraints, Graphs, Algebra, Logic, and Complexity*
}

\author{
Moshe Y. Vardi ${ }^{1}$ \\ 1 Department of Computer Science \\ Rice University, Houston, TX 77005, USA \\ vardi@cs.rice.edu.com
}

\begin{abstract}
A large class of problems in AI and other areas of computer science can be viewed as constraintsatisfaction problems. This includes problems in database query optimization, machine vision, belief maintenance, scheduling, temporal reasoning, type reconstruction, graph theory, and satisfiability. All of these problems can be recast as questions regarding the existence of homomorphisms between two directed graphs. It is well-known that the constraint-satisfaction problem is NP-complete. This motivated an extensive research program into identify tractable cases of constraint satisfaction.

This research proceeds along two major lines. The first line of research focuses on non-uniform constraint satisfaction, where the target graph is fixed. The goal is to identify those target graphs that give rise to a tractable constraint-satisfaction problem. The second line of research focuses on identifying large classes of source graphs for which constraint-satisfaction is tractable. We show in how tools from graph theory, universal algebra, logic, and complexity theory, shed light on the tractability of constraint satisfaction.
\end{abstract}

1998 ACM Subject Classification F.1.3 Complexity Measures and Classes

Keywords and phrases constraint satisfaction, NP completeness, dichotomy

Digital Object Identifier 10.4230/LIPIcs.FSTTCS.2011.3

\section{References}

1 P.G. Kolaitis and M.Y. Vardi. A logical approach to constraint satisfaction. In Complexity of Constraints, Lecture Notes in Computer Science 5250, pp. 125-155, Springer, 2008.

\footnotetext{
* Work supported in part by NSF grants CCF-0728882, and CNS 1049862, by BSF grant 9800096, and by gift from Intel. 